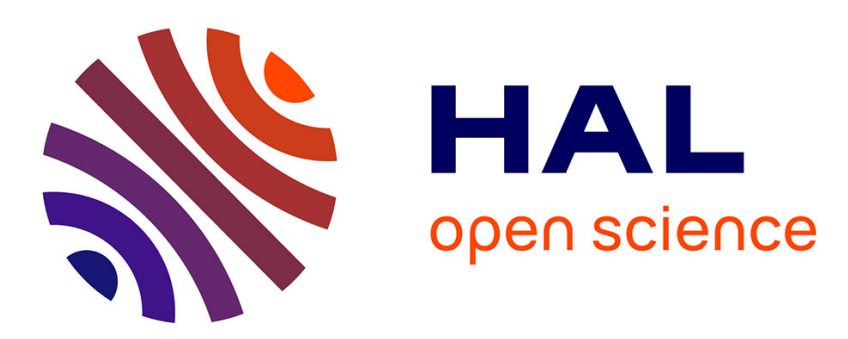

\title{
A model of neural mechanisms in monocular transparent motion perception
}

Florian Raudies, Pierre Bayerl, Heiko Neumann

\section{To cite this version:}

Florian Raudies, Pierre Bayerl, Heiko Neumann. A model of neural mechanisms in monocular transparent motion perception. Deuxième conférence française de Neurosciences Computationnelles, "Neurocomp08", Oct 2008, Marseille, France. hal-00331556

\section{HAL Id: hal-00331556 https://hal.science/hal-00331556}

Submitted on 17 Oct 2008

HAL is a multi-disciplinary open access archive for the deposit and dissemination of scientific research documents, whether they are published or not. The documents may come from teaching and research institutions in France or abroad, or from public or private research centers.
L'archive ouverte pluridisciplinaire HAL, est destinée au dépôt et à la diffusion de documents scientifiques de niveau recherche, publiés ou non, émanant des établissements d'enseignement et de recherche français ou étrangers, des laboratoires publics ou privés. 


\title{
A MODEL OF NEURAL MECHANISMS IN MONOCULAR TRANSPARENT MOTION PERCEPTION
}

\author{
F. Raudies, P. Bayerl, and H. Neumann \\ Institute of Neural Information Processing, University of Ulm \\ Oberer Eselsberg, D-89069 Ulm, Germany \\ email: florian.raudies@uni-ulm.de,pierre.bayerl@alumni.uni-ulm.de,heiko.neumann@uni-ulm.de
}

\begin{abstract}
Transparent motion is perceived when multiple motions are presented in the same part of visual space which moves in different directions. Several psychophysical as well as physiological experiments have studied the conditions under which motion transparency occurs. Few model mechanisms have been proposed to segregate multiple motions. We present a novel neural model which investigates the necessary mechanisms underlying initial motion detection, the required representations for velocity coding, and the integration and segregation of motion stimuli. The model extends a previously developed architecture for dorsal pathway computations, particularly, in cortical areas V1, MT, and MST, emphasizing the role of feedforward cascade processing and feedback from higher to earlier stages for selective feature enhancement and tuning. Our results demonstrate that the model reproduces several psychophysical findings using random dot stimuli. Moreover, the model is able to process transparent motion in real-world sequences of 3D scenes.
\end{abstract}

\section{KEY WORDS}

transparent motion, neural model, motion integration, area MT, area MST, feedback.

\section{Introduction}

Displays of overlaid movement patterns moving in different directions are perceived as transparent motion under certain conditions. In psychophysical experiments random dot and plaid stimuli have been utilized to investigate the ability of humans to segregate such motions. Physiological experiments help to reveal the underlying neuronal mechanisms leading to perceptual representations of transparent motion in primates. Critical stimulus parameters studied were the influence of paired versus unpaired moving dots, the impact of noise, the display conditions of multiple coherent motions and coherent vs random motions (in overlays or separate sectors), and the difference angle between different overlaid motions $[20,4,6]$. In $[12,4]$ the acuity to discriminate between dots moving in opposite directions which are located in stripes of varying width have been investigated. Categorical differences were observed such that transparent motion is perceived for small stripes while for wide stripes coherent motion alternating in opposite direc-

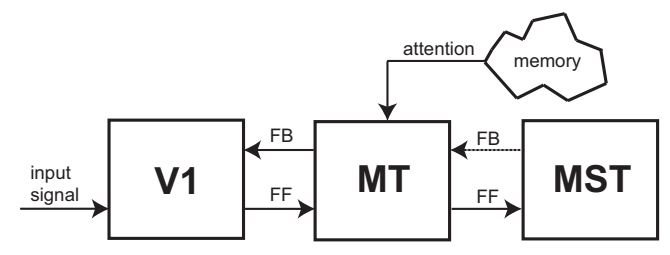

Figure 1. Model overview showing the cascade of different model areas involved and their interaction.

tions is perceived. Attention deployed to certain motion directions can improve human performance in segregating up to 6-8 simultaneously presented transparent motion directions in comparison to 2-3 directions in unattended stimuli [8]. Such an improvement has been attributed to a selective tuning mechanism that improves selectivity of visual processing [19]. The discrimination of motion directions in regular displays has been investigated demonstrating that motion directions which differ less than $10 \mathrm{deg}$ are perceived in a compromise direction [21].

Here, we propose a novel neural model of motion detection and integration that builds upon previous work reported in [2]. The recurrent interaction between model areas, namely V1, MT and MST in the dorsal pathway, and the feature representations at the different stages are investigated. As a main contribution, in order to enable the perception of transparent motion, it is proposed how multiple velocities in a log-polar sampled speed-direction space can be represented at individual spatial locations and how the neural interactions are defined. Other computational approaches, including computer vision algorithms, attempt to optimize representations with respect to single velocities and investigate the necessary conditions in representing multiple motions corresponding with transparent layers [20, 7, 23]. Here, we suggest local competitive centersurround interaction between responses in velocity space that leads to mutual competition or coexistence of multiple motion representatives depending on their separation in velocity space. Unlike other modeling attempts such as, e.g., [13] or [24] we propose a scheme that allows to process motion of both opaque surfaces as well as to detect and integrate transparent motion patterns in a unified architecture. Also, the model successfully deals with artificial as well as with real image sequences. 


\section{Neural Computational Mechanisms}

The model architecture for motion processing is composed of three hierarchically organized areas allocated along the dorsal pathway, namely V1, MT, and MST. The areas are linked bidirectionally to build a recurrent scheme of feedforward (FF) / feedback (FB) interaction (see Fig.1). Initial motion detection is achieved by extended Reichardt detectors [17] whereas Gabor filter responses are used for spatiotemporal correlation. Each model area is described by a hierarchical three-level cascade of processing stages. The first stage (eq. 2) consists of an initial (linear or non-linear) filtering stage and subsequent velocity blurring which leads to an activity integration and suppression of small activations. At the second stage a modulatory coupling of FB signals enhances driving input activations (eq. 3). This mechanism ensures that FB signals from stages at higher processing levels cannot generate any activation in lower levels without coexisting non-zero bottom-up activation. The spatial spread of FB connections is formally described by a Gaussian kernel $\Lambda^{F B}$. In the present model simulations we set $\Lambda^{F B}=\delta$ for computational reasons. The third stage realizes an activity normalization achieved by a stage of center-surround shunting inhibition (eq. 4). Model MT integration cells were proposed to generate local velocity space representations which are subsequently processed by large-field integration cells (at MSTd) and contrast cells (at MSTl) which, in turn, may enhance compatible input activities via FB. Model MST cells were used only in one experiment in order to investigate the global segregation of transparent motion configurations. ${ }^{1}$ A top-down signal has been incorporated that enters into model stage MT. This is intended to investigate the influence of attention when it is deployed to a pre-selected motion feature (compare Fig.1).

In this model architecture the stage of shunting centersurround competition is organized to allow multiple activations in velocity space at individual spatial locations in model MT. The topography of the velocity space representation samples speed and direction features in a log-polar fashion [15]. Activities in velocity space are further processed by center-surround mechanisms using filter kernels $\Lambda^{+}$and $\Lambda^{-}$. Here, we utilize Gaussian kernels for the interaction of activities which vote for velocities. Through this competition the activation corresponding to the center filter kernel is enhanced, while others are suppressed.

Neural activation is denoted in formal terms by utilizing single compartment cell models with gradual activation dynamics [10]. The cell activity is described by the membrane potential subject to changes given external excitatory and inhibitory synaptic input. This leads to the dynamics denoted by the following membrane equation (assuming zero resting level)

$$
C \dot{x}=-A \cdot x-\left[x-E_{+}\right] \cdot I_{+}-\left[x-E_{-}\right] \cdot I_{-},(1)
$$

${ }^{1}$ Model area MST is supplied to investigate global integration of multiple velocities present at individual spatial locations. The computation has been simplified such that MT only feeds MST pattern motion cells via FF signal integration. where $x$ denotes the activation as a function of $t, I_{+,-}$denote time dependent conductances corresponding to the exitatory and inhibitory input, respectively, $E_{+,-}$denote reversal potentials, and $C$ is the capacity of the membrane [11]. The three-level cascade which describes the computations in each model area, namely V1, MT, and MST, is formally described by variants of the membrane equation in eq. 1 . We get

$$
\begin{aligned}
\dot{x}^{(1)}= & -x^{(1)}+\left[x^{F F_{i n}}\right]^{\beta} * \Lambda^{b} \\
\dot{x}^{(2)=} & -x^{(2)}+x^{(1)} \cdot\left[1+\gamma\left(x^{F B_{i n}} * \Lambda^{F B}\right)\right], \\
\dot{x}^{(3)=} & -A x^{(3)}+\left(B-D x^{(3)}\right)\left(x^{(2)} * \Lambda^{+}\right) \\
& -\left(C+E x^{(3)}\right)\left(x^{(2)} * \Lambda^{-}\right)
\end{aligned}
$$

with $\beta=2, \gamma=10^{3}, \Lambda^{F B}, A=10^{-2}, B=1, C=0$, $D=0, E=1$. For the velocity blur, denoted by the kernel $\Lambda^{b}$, separable Gaussian filters were used with $\sigma_{s}=0.5 \mathrm{px}$ (speed) and $\sigma_{d}=1.0 \mathrm{px}$ (direction). The center-surround interaction mechanism again uses separable kernels with $\sigma_{s}^{+}=0.25 \mathrm{px}$ and $\sigma_{d}^{+}=0.5 \mathrm{px}$ (center) and $\sigma_{s}^{-}=0.75 \mathrm{px}$ and $\sigma_{d}^{-}=4.0 \mathrm{px}$ (surround). Velocities for 6 speeds and 32 directions are represented. ${ }^{2} x^{F F_{i n}}$ and $x^{F B_{i n}}$ denote activations of the driving forward stream and the modulatory feedback stream, respectively, and $*$ denotes the convolution operation. In our simulations all equations were solved at steady-state.

For the driving feedforward processing a spatial integration with a ratio of 1:5 between areas V1 and MT is assumed. This relates to eq. 2 in the processing cascade. The feedback mechanism is predictive such that an offset of the spatial location of the modulatory interaction is incorporated that depends on the particular velocity ([2]). Processing of a full FF/FB cycle is assumed to take approximately $20 \mathrm{~ms}$ as indicated by measurements of V1-MT interactions in monkey [22] (compare with [2]).

\section{Results}

The model has been probed with different test stimuli which consist of different motion patterns with transparent surface arrangements. Here, grating acuity has been investigated [12, 5]. In addition, the influence of attention is investigated to demonstrate increased performance for selected movements [8]. To comply with the psychophysical experiments random dot kinematograms of size $256 \times 256 \mathrm{px}, 10 \%$ dot density, and dots with a diameter of approximately $7 \mathrm{px}$ (interlaced) were used. In order to demonstrate the generic computational competency, the model was also probed with test sequences from threedimensional scenes and test scenes with opaque surface arrangements.

Motion segregation. The segregation of transparent motion patterns has been investigated psychophysically by

\footnotetext{
${ }^{2}$ For random dot kinematograms used as input only 3 speed values were employed, and parameters of the center-surround mechanism were adapted to this new configuration (' $\sigma_{s}^{+}=0$ ' $\mathrm{px}$ and $\sigma_{s}^{-}=0.5 \mathrm{px}$ ).
} 


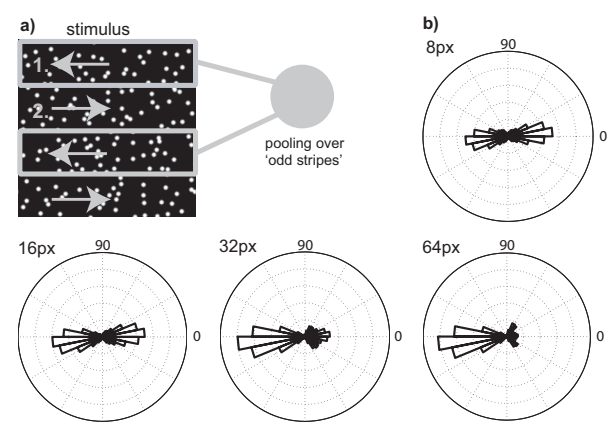

Figure 2. Varying grating acuity results in a transparent or coherent motion percept depending on the stripe widths. a) Input stimuli. b) Velocity distribution for activations summed over 'odd' stripes in model area MT (5th iteration).
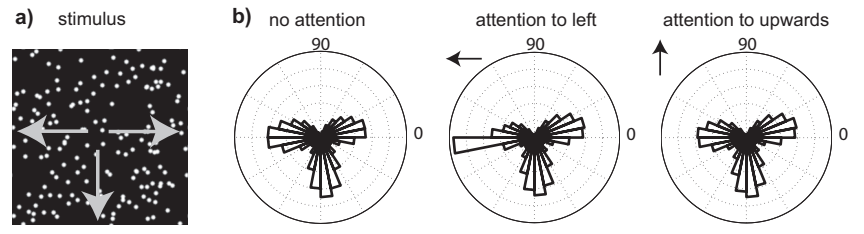

Figure 3. Attentional priming selectively amplifies initially detected motion directions. a) Input stimuli with three motions overlaid. b) Activities plotted in direction histograms. In the unattended case, different motion directions are represented by a population of velocity sensitive model MT cells. Selective deployment of attention to leftward motion enhances the corresponding activities, while attention to upward motion does not influence the activity distribution. All activations shown are for the 6th iteration when the attention signal is deployed during the 5 th iteration.

using stimuli consisting of stripes of a given width which contain coherent movements where direction alternates between pairs of stripes $[12,5,7]$. Stripe widths in the employed random dot kinematograms varied from 8 to $64 \mathrm{px}$. Figure 2 shows cell activations in MT velocity spaces as a function of the specified stripe widths. In case of a stripe width of $8 \mathrm{px}$ fully transparent motion is represented in area MT as signaled by the bimodal distribution of activity. Increasing the stripe width the distribution of activities in velocity space gradually change into a more unimodal direction distribution for model MT cells.

Attentional priming. It has been demonstrated in [14] that the number of transparent motion directions is limited to two or three different directions displayed simultaneously. To overcome this limitation, selective attention may help to focus processing on the relevant stimulus components in a specific task [19]. In a more recent study, [8] have shown that the number of distinguishable transparent motion directions can indeed be significantly increased by attending to pre-selected directions. Here, we demonstrate computationally how the influence of a signal generating an attentional prime for a selected motion direction can help to improve direction selectivity. In the proposed model an attention signal is assumed to be deployed after stabilization of the motion percept which is assembled by a proper activation in working memory and delivered via a top-down excitatory feedback signal. It should be emphasized that such an attention signal utilizes the same circuits as the inter-areal sensory $\mathrm{FF} / \mathrm{FB}$ interactions at earlier stages of cortical processing (compare [3]).

In computational terms the feature attention signal is introduced at the fifth iteration of simulating the network dynamics with a strength $5 / C_{F B}$ and spreads with a spatial uncertainty (for directions and speeds) using a Gaussian with $\sigma_{a t t}=0.25$. Again the previously described random dot kinematograms served as input. Figure 3 shows that the attentional priming signal can enhance activation at area MT to enforce a decision with respect to a given motion direction. In a control experiment, we show that this is only the case, when the motion direction was initially present in the input to generate an activation in the corresponding velocity space representation.

Three-dimensional surface arrangements and realistic scenes. In order to demonstrate that the model is also capable to process sequences from realistic scenes, slanted transparent surfaces were rendered by using the POV-Ray ray-tracer. In Fig. 4 the processing results show that the overlaid motion generated by the transparent region can be properly segregated. In addition, we probed the model by using image sequences which have been used to benchmark computer vision algorithms. Consider, for example, the configuration shown in Fig. 5. Here the background (facial image) is translating to the left while the earth globe (clouds) rotates clockwise. Again, iterative computation is done at the stages of V1 and MT. In the central circular patch covered by the cloud image the proposed neural mechanisms robustly generate multi-peaked velocity representations at same spatial locations. Model MT cells (defined in velocity space) feed their activities to pattern cells in model MSTd. Such cells are selective to canonical flow patterns such as expansion, contraction as well as clockwise and anti-clockwise rotation, while a different population is selective to planar translational motion [9]. The results show that the two transparent motion patterns generate dominant activations in those cells with respective pattern selectivity. We therefore suggest that MT-MST interaction stabilizes velocity estimates and contributes to the segmentation of transparent motion patterns. Finally, we investigated the processing of test sequences (e.g. Yosemite flight through with clouds) which contain opaque object surfaces only (results not shown). The successful processing thus demonstrates that the scope of the model is broad enough to reliably deal with various surface configurations.

Coherent motion and decision making. Finally, we tested the sensitivity of the proposed mechanism against noise influences and evaluated the ability to decide on the 
such that $\eta$ expresses a significance ratio for the difference between $a_{+}$and $a_{-} . U(\{0,1\})$ denotes a binary random variable with probability 0.5 . Fig. $6 \mathbf{b}$ ) shows results of the decision making process for different threshold settings given extrinsic as well as intrinsic noise (500 trials). The data was fitted by a cumulative Weibull distribution with two parameters $\alpha$ (threshold) and $\beta$ (steepness). By increasing the threshold parameter $\eta$ in eq. 6 the decision function is shifted to the left. For comparison, we have included results from monkey experiments by [18] (their Fig.3A).

\section{Discussion and Conclusion}

The processing of motion in a situation where two or more transparent surfaces move transparently over each other is still a big challenge for modelers of biological as well as computational motion perception [20]. In this paper, we propose a neural model for the perception of transparent motion. The proposal makes several new main contributions, namely (i) the reliable processing of transparent motion stimuli by suggesting an on-center/off-surround interaction mechanism in a velocity space representation of model MT, (ii) the incorporation of a log-speed/direction representation incorporating the above center-surround interactions, (iii) the augmentation of an attentional priming signal utilizing the circuitry of bottom-up and top-down signal flow for stabilizing motion feature detection and integration, and (iv) the incorporation of pattern motion cells in model MSTd to segregate global transparent motions into separate surfaces.

Several investigators analyzed whether the representation of two motions at the same location is sufficient for the perception of transparent motion and whether such input configurations are processed in parallel [6]. Previous models have been suggested to account for processing of transparent motion. For example, [16] proposed a twostage FF model of V1 and MT processing. In this model motion energy is detected and rectified at the level of V1 [1] and subsequently integrated at the stage of MT assuming opponent direction inhibition within subfields. We also utilize inhibition between subunits and also emphasize the role of shunting inhibition. Unlike their model, however, we propose that opponent inhibition operates on the full velocity space and the activation represented therein. This enables the selective tuning to multiple motions and allows the fusion of motion directions along similar directions represented by a compromise direction. Furthermore, the iterative interaction of FF and FB activities demonstrates how initially ambiguous and unreliable estimates can be disambiguated, and how attentional priming can bias and selectively enhance activations. The model of [24] proposed a network of orthogonally oriented extended Reichardt detectors [17]. For different input displays with two and multiple transparent motions (global) motion vector histograms have been generated using the detector network. The number of individual maxima in the distributions is taken as indicator of perceptual separability of transparent motions. These findings reveal important parameters of tuning properties of the basic mechanisms for motion detection. Recent work by [7] have shown, however, that multimodal velocity distributions (histograms) are not sufficient to indicate transparency percepts (compare also our first computational study depicted in Fig.2). We have emphasized how different motion directions can be segregated computationally based on generic neural interactions using the neural representation of velocities in area MT. When these velocities are integrated and further processed at the stage of MSTd, coherent motions can be segregated and represented at the same spatial location. Another model by [13] proposes a generalized structure tensor which is defined as the tensor product of the N-th order partial space-time derivatives of the input stimulus. The rank of these generalized tensors determines the number of motion directions. In their model, to identify those motions, the input pattern is transformed into Fourier domain, where motion is represented as plane/line which is then mapped by a projective transform. Using the duality of the projective plane gives the velocity or set of possible velocities. We emphasize the neural mechanisms involved in this processing. At the moment, it remains how the gradual changes in perceiving transparent and non-transparent motion in parallel stripes of opposite motions can be generated using global Fourier transform or how attention can be incorporated in such a model. We have proposed a testable mechanism that demonstrates successfully motion detection and integration for transparent as well as opaque surfaces. Feedback signal enhancement and feedback that is deliberately supplied to focus attention on a particular motion direction is handled using the same model neural circuits.

Psychophysical studies report that the ability to perceive transparent motions (i) depends on the angle between the discriminating motion directions, and (ii) increases with higher speeds of motions $[12,8]$. Both findings directly refer to the properties of the velocity space and the shunting mechanism employed in the suggested model. For small difference angles and small speeds the resulting activations mutually compete and these result in low activation. In contrast, motions with a high discriminating angle or speed will be represented with a stronger activation. Thus the model makes the prediction of a systematic dependence between angular difference, motion speeds and the acuity to perceive transparent motion. We have proposed a centersurround shunting mechanism defined in velocity space and demonstrated that this computational mechanism is robust against different noise influences. A decision-making process has been simulated in which an observer selects a motion direction between two opposite directions (2AFC task) based on the ratio of velocity responses pooled over a spatial region. The fitted psychometric functions for different threshold settings correspond to the results of a direction discrimination task reported by [18]. This demonstrates that the model representations generated provide results comparable with animal behavior in decision-making. 
In all, we presented a neural mechanism of transparent motion processing based on model areas V1, MT, and MST, and their inter-areal as well as intra-areal interactions extending the model of [2]. With these extensions the detection, representation, and processing of transparent motion stimuli as well as the perception of motion of opaque surfaces can be explained in a single framework. Our model can explain psychophysical findings concerning grating acuity [5] and attentional priming [12, 8]. We claim that such mechanisms will help to steer the learning to improve performance in speed and direction discrimination tasks. This will be investigated in the future. In addition, further work will also focus on interactions with the form channel to increase alignment acuity [5].

\section{Acknowledgements}

This research has been supported in part by grants from the European Union (EU FP6 IST Cognitive Systems Integrated project Decisions-in-Motion, project no. 027 198), the German Ministry of Research and Technology (BMBF, Brain Plasticity and Perceptual Learning, project no. 01GW0763), and a scholarship to F.R. from the Graduate School of Mathematical Analysis of Evolution, Information and Complexity at Ulm University. We thank Pierre Kornprobst (Odyssee lab, INRIA) for kindly providing the image sequence used in Fig.5.

\section{References}

[1] E.A. Adelson and J.R. Bergen. Spatiotemporal energy models for the perception of motion. J. of Opt. Soc. of Amercia, Series A, 2:284-299, 1985.

[2] P. Bayerl and H. Neumann. Disambiguation visual motion through contextual feedback modulation. Neural Comp., 16:2041-2066, 2004.

[3] P. Bayerl and H. Neumann. A neural model of feature attention in motion perception. BioSys., 89:208-215, 2007.

[4] O. Braddick and N. Qian. The organization of global motion and transparency. In J. Zanker, J. Zeil (eds.) Motion Vision: Computational, neural, and ocological constraints, Springer Verlag, Heidelberg, 2001, pages $85-112$.

[5] D. Burr, S. McKee, and C.M. Morrone. Resolution for spatial segregation and spatial localization by motion signals. Vis. Res., 46:932-939, 2006.

[6] W. Curran, P.B. Hibbard, and A. Johnston. The visual processing of motion-defined transparency. Proc. Roy. Soc. of London B, 274:1049-1056, 2007.

[7] S. Durant, A. Donoso-Barrera, S. Tan, and A. Johnston. Moving from spatially segregated to transparent motion: a modelling approach. Biology Letters, 2:101-105, 2006.

[8] F.M. Felisberti and J.M. Zanker. Attention modulates perception of transparent motion. Vis. Res., 45:25872599, 2005.

[9] M.S.A Graziano, R.A. Andersen, and R. Snowden. Tuning of mst neurons to spiral motions. J. of Neuroscience, 14:54-67, 1994.

[10] A.V.M. Herz, T. Gollisch, C.K. Machens, and D. Jaeger. Modeling single-neuron dynamics and computations: a balance of details and abstraction. Science, 314:80-85, 2006.

[11] A.L. Hodkin and A.F. Huxley. A quantitative description of membrane current and its application to conduction and excitation in nerve. J. of Phys., 117:500544, 1952.

[12] D.R. Mestre, G.S. Masson, and L.S. Stone. Spatial scale of motion segmentation from speed cues. Vis. Res., 41:2697-2713, 2001.

[13] C. Mota, M. Dorr, I. Stuke, and E. Barth. Categorization of transparent-motion patterns using the projective plane. J. Comp. and Inf. Sc., 5(2):129-40, 2004.

[14] J.B. Mulligan. Motion transparency is restricted to two planes. In Inv. Ophth. Vis. Sci. (suppl.), 33, 1049, 1992.

[15] H. Nover, C.H. Anderson, and G.C. DeAngelis. A logarithmic, scale-invariant representation of speed in macaque middle temporal area accounts for speed discrimination performance. J. of Neuroscience, 25(24):10049-10060, 2005.

[16] N. Qian, R.A. Anderson, and E.A. Adelson. Transparent motion perception as detection of unbalanced motion signals. iii. modeling. J. of Neuroscience, 14(12):7381-7392, 1994.

[17] W. Reichardt. Evaluation of optical information by movement detectors. J. of Comp. Phys. A, 161:533547, 1987.

[18] J.D. Roitman and M.N. Shadlen. Response of neurons in the lateral intraparietal area during a combined visual discrimination reaction time task. J. of Neuroscience, 22(21):9475-9489, 2002.

[19] A.L. Rothenstein and J.K. Tsotsos. Attention links sensing to recoginition. Im. and Vis. Comp., 26:114126, 2008.

[20] R.J. Snowden and F.A.J. Verstraten. Motion transparency: making models of motion perception transparent. Trends in Cogn. Sci., 3:369-377, 1999.

[21] S. Treue, K. Hol, and H.-J. Rauber. Seeing multiple directions of motion - physiology and psychophysics. Nature Neuroscience, 3(3):270-276, 2000.

[22] S. Vanni, M. Dojat, J. Warnking, C. Delon-Martin, C. Segebarth, and J. Bullier. Timing of interactions across the visual field in the human cortex. NeuroImage, 21:818-828, 2004.

[23] O. Watanabe and M. Kikuchi. Nonlinearity of the population activity to transparent motion. Neural Networks, 18:15-22, 2005.

[24] J.M. Zanker. A computational analysis of separating motion signals in transparent random dot kinematograms. Spat. Vis., 18(4):431-445, 2005. 